ARTICLE

Received 12 Jan 2016 | Accepted 8 Apr 2016 | Published 25 May 2016

DOI: $10.1038 /$ ncomms11565

OPEN

\title{
Molecular evidence of functional progesterone withdrawal in human myometrium
}

\author{
Lubna Nadeem ${ }^{1}$, Oksana Shynlova ${ }^{1,2}$, Elzbieta Matysiak-Zablocki ${ }^{1}$, Sam Mesiano ${ }^{3}$, Xuesen Dong $^{2,4, \star}$ \\ $\&$ Stephen Lye $\mathrm{e}^{1,2,5, *}$
}

Progesterone suppresses uterine contractility acting through its receptors (PRA/B). The mechanism by which human labour is initiated in the presence of elevated circulating progesterone has remained an enigma since Csapo first theorized of a functional withdrawal of progesterone in 1965. Here we report that in vitro progesterone-liganded nuclear PRB forms a complex including JUN/JUN homodimers and P54 nrb/Sin3A/HDAC to repress transcription of the key labour gene, Cx43. In contrast, unliganded PRA paradoxically activates $\mathrm{C} \times 43$ transcription by interacting with FRA2/JUND heterodimers. Furthermore, we find that while nuclear progesterone receptor (PR) is liganded during human pregnancy, it becomes unliganded during both term and preterm labour as a result of increased expression of the progesterone-metabolizing enzyme $20 \alpha \mathrm{HSD}$ and reduced nuclear progesterone levels. Our data provide a mechanism by which human labour can occur in the presence of elevated circulating progesterone and suggests non-metabolizable progestogen might represent an alternative new therapeutic approach to preterm birth prevention.

\footnotetext{
${ }^{1}$ Lunenfeld Tanenbaum Research Institute, Mount Sinai Hospital, Toronto, Ontario, Canada M5T 3H7. ${ }^{2}$ Department of Obstetrics \& Gynecology, University of Toronto, Toronto, Ontario, Canada M5G 1E2. ${ }^{3}$ Department of Reproductive Biology, Case Western Reserve University, Cleveland, Ohio 44106-5034, USA.

${ }^{4}$ Department of Urologic Sciences, Vancouver Prostate Centre, University of BC, Vancouver, British Columbia, Canada V6H 3Z6. ${ }^{5}$ Department of Physiology University of Toronto, Toronto, Ontario, Canada M5S 1A1. * These authors contributed equally to this work. Correspondence and requests for materials should be addressed to S.L. (email: Iye@lunenfeld.ca).
} 
M ore than 85 years after the discovery of progesterone (P4) by Corner and Allen ${ }^{1}$, we have limited understanding of its molecular role in pregnancy maintenance and the initiation of labour. Progesterone suppresses spontaneous uterine contractility during pregnancy and, in most mammals, a fall in systemic P4 levels ('progesterone withdrawal') is required for the initiation of labour at term. However, in humans, labour occurs in the presence of elevated circulating levels of $\mathrm{P} 4$, leading Csapo to propose the 'functional $\mathrm{P} 4$ withdrawal' theory ${ }^{2,3}$ though the mechanisms by which this is achieved have yet to be defined.

Over many decades researchers have proposed hypotheses to explain the functional withdrawal of $\mathrm{P} 4$, including, the sequestration of active $\mathrm{P} 4$ by corticosteroid-binding globulin ${ }^{4}$, a decrease in active $\mathrm{P} 4$ metabolite levels ${ }^{5}$, changes in the ratio of progesterone receptor (PR) isoforms ${ }^{6}$ or transcriptional co-activators and/or repressors ${ }^{7}$, a functional oestrogen activation ${ }^{8}$ and inflammation resulting in NF- $\mathrm{KB}$-mediated PR repression ${ }^{9}$. While there is a lack of compelling evidence to support any of these hypotheses, the theory of a 'functional P4 withdrawal' remains valid since disruption of P4 signalling by the PR antagonist RU486 at any stage of pregnancy results in myometrial contractions and labour in mice ${ }^{10}$, rats ${ }^{11}$ and women ${ }^{12}$, while the treatment of pregnant rodents with $\mathrm{P} 4$ delays myometrial contractions and parturition ${ }^{13}$

One of the hypotheses postulates that the differential expression of the progesterone receptor isoforms, PRA and PRB contributes to a functional withdrawal of $\mathrm{P} 4$ and the onset of labour ${ }^{6}$. PRB is the full-length receptor while PRA lacks the 164 amino acid AF3-activation domain at the $\mathrm{N}$ terminus ${ }^{14,15}$. We have suggested that PRB dominates throughout pregnancy and mediates myometrial quiescence, while during labour an increase in the PRA:PRB ratio leads to the functional suppression of PRB and the induction of labour ${ }^{6,16,17}$. However, until now, the specific mechanisms by which these isoforms contribute to labour onset remain unclear.

Labour requires increased expression of genes (for example, GJA1(Cx43), PTGS2(COX2), OXTR and NFKB2) that mediate myometrial 'activation' and optimal responsiveness to uterotonic agonists such as stimulatory prostaglandins and oxytocin. Among these labour genes, the gap junction protein $\mathrm{Cx} 43$ plays an essential role in the initiation of term or preterm labour through cell-cell coupling and generation of synchronous myometrial contractions ${ }^{18-23}$. The $C x 43$ gene is regulated by the AP-1 (Fos/Jun) family of transcription factors that function as either Jun/Jun homodimers or Fos/Jun heterodimers ${ }^{24,25}$. We have shown that Fos/Jun heterodimers are strong inducers of $\mathrm{Cx} 43$ transcription compared with Jun/Jun homodimers ${ }^{26,27}$ and that P4, acting through PRs, represses $\mathrm{Cx} 43$ transcription. The physical interaction between PRs and cJun results in the recruitment of PR-p54 ${ }^{\text {nrb }} / \mathrm{mSin} 3 \mathrm{~A} / \mathrm{HDAC}$ transcriptional repressor complex to the AP- 1 consensus site in the $\mathrm{Cx} 43$ promoter ${ }^{28,29}$.

We now demonstrate that during pregnancy progesterone confers transcriptional repression of myometrial $\mathrm{Cx} 43$ through the formation of PRB-JUN/JUN homodimer complex, whereas during labour an increased expression of FOS proteins favours binding of PRA to the $\mathrm{Cx} 43$ promoter. Remarkably, during labour nuclear PRA is unliganded, even in the presence of elevated circulating P4 levels and that in this unliganded state, PRA acts as a transcriptional activator (rather than repressor) of $\mathrm{Cx} 43$. Finally, we provide evidence that the loss of binding of P4 to PRA is due to a reduction in intracellular P4 levels in the myometrium as a result of increased expression of the metabolizing enzyme, $20 \alpha$ hydroxysteroid dehydrogenase $(20 \alpha H S D)$. Altogether, these data provide a mechanistic basis for the functional withdrawal of P4 in human labour.

\section{Results}

PR differentially interact with AP-1 transcription factors. We previously showed that AP-1 proteins modulate transcription of Cx43 (ref. 26). Since an increase in the PRA:PRB ratio has been suggested to mediate a functional withdrawal of $\mathrm{P} 4$, we investigated the interaction of $\mathrm{PR}$ isoforms with the AP-1 proteins. To differentiate between two PR isoforms, we used human myometrial hTERT-HM cell lines stably expressing Flagtagged PRA and PRB as described earlier ${ }^{30}$. The endogenous expression of AP-1 proteins in this cell line (Supplementary Fig. 1) and human myometrium (labouring and non-labouring) is shown in Supplementary Fig 2. hTERT-HM cells were stimulated with P4 and subjected to PLA analysis to characterize interactions between AP-1 proteins and PR isoforms (Fig. 1a). Compared with PRA, PRB showed stronger interaction with cJun and JunB proteins, similar affinity towards JunD and cFos and weaker interaction with Fra1 or Fra2 proteins; both receptors exhibited minimal interaction with FosB (Fig. 1a). Quantitation of PLA indicated that there was a significant increase $(P<0.05)$ in the affinity of PRA (relative to PRB) to Fra1, Fra2 proteins in a ligand-independent manner (Fig. 1b, Supplementary Fig. 3). We confirmed these interactions using in vitro co-immunoprecipitation assay in Syrian hamster myometrial (SHM) cells (Supplementary Fig. 4).

Next, we examined the interaction between PRA/B and the co-repressor proteins $\mathrm{p} 54^{\mathrm{nrb}} / \mathrm{mSIN} 3 \mathrm{~A}$ which mediate $\mathrm{Cx} 43$ suppression. PRB showed strong affinity for both co-repressors, whereas PRA had minimal interaction with $\mathrm{p} 54^{\mathrm{nrb}}$ or mSIN3A (Fig. 1a,b).

AP-1 dimers determine PR modulation of cx43 transcription. We next determined the functional impact of the interactions between AP-1 dimers and PR isoforms on $\mathrm{Cx} 43$ transcription using SHM cells co-transfected with a Cx43 luciferase promoter (pCx300-luc containing the proximal AP-1 site, Supplementary Fig. 5) ${ }^{26,31}$, PRA or PRB isoforms and either JUNB/JUND homodimers or FRA2/JUND heterodimers (FRA2 is the predominant nuclear FOS protein during labour; Supplementary Fig. 2). Our data indicate that the AP-1 dimer composition determines the modulation of $\mathrm{Cx} 43$ transcription by the $\mathrm{PR}$ isoforms. Thus, in the presence of JUNB/JUND homodimers both PRA and PRB repress transcription of $\mathrm{Cx} 43$, while in the presence of FRA2/JUND heterodimers PRA (but not PRB) activates $\mathrm{Cx} 43$ transcription $(P<0.05$; Fig. 2a). A two base-pair mutation of the AP-1 consensus sequence from TGAGTCA to TGAGTtg (pCx300(m)-luc, Supplementary Fig. 5) resulted in the abrogation of PR-JUNB/JUND mediated repression and PRAFRA2/JUND mediated induction of $\mathrm{Cx} 43$ promoter activity (Fig. 2b). The activation of $\mathrm{Cx} 43$ transcription by PRA-FRA2/ JUND was further enhanced using a $\mathrm{Cx} 43$ promoter construct that contained both proximal and distal AP-1 sites (pCx1686-luc, Supplementary Fig. 5, Fig. 2c). Importantly, in the presence of progesterone PRA no longer activated $\mathrm{Cx} 43$ transcription (Fig. 2d).

Myometrial PRs are unliganded during labour. Our unexpected in vitro finding that unliganded PRA acts as a transcriptional activator of Cx43 led us to investigate whether PRA is unliganded during human labour when $\mathrm{Cx} 43$ transcription is greatly increased. Using PLA, we confirmed that before the onset of labour PRs are liganded to P4 in the human myometrium. However, despite the abundance of circulating P4, PRs are unliganded during labour (Fig. 3a). We sought to validate this remarkable observation in an animal model in which a systemic P4 withdrawal leads to the onset of labour. As predicted, PLA 
a
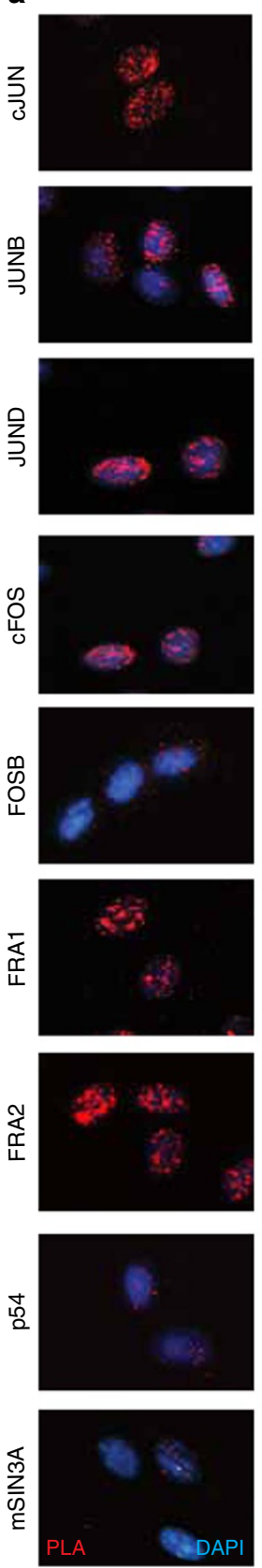
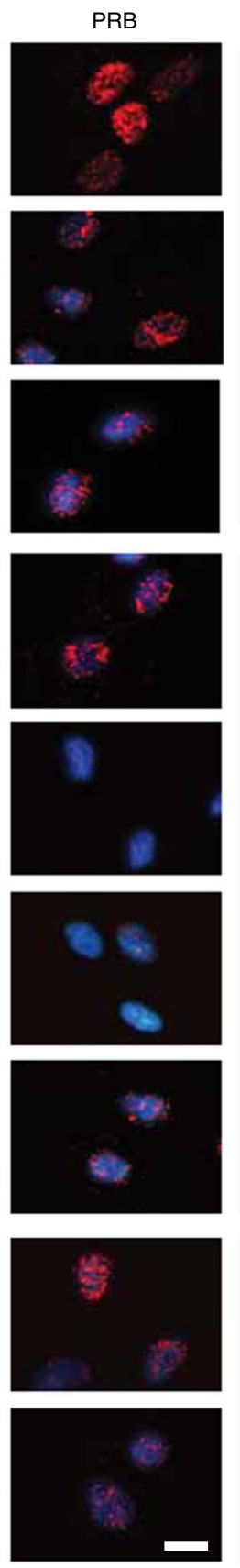

b
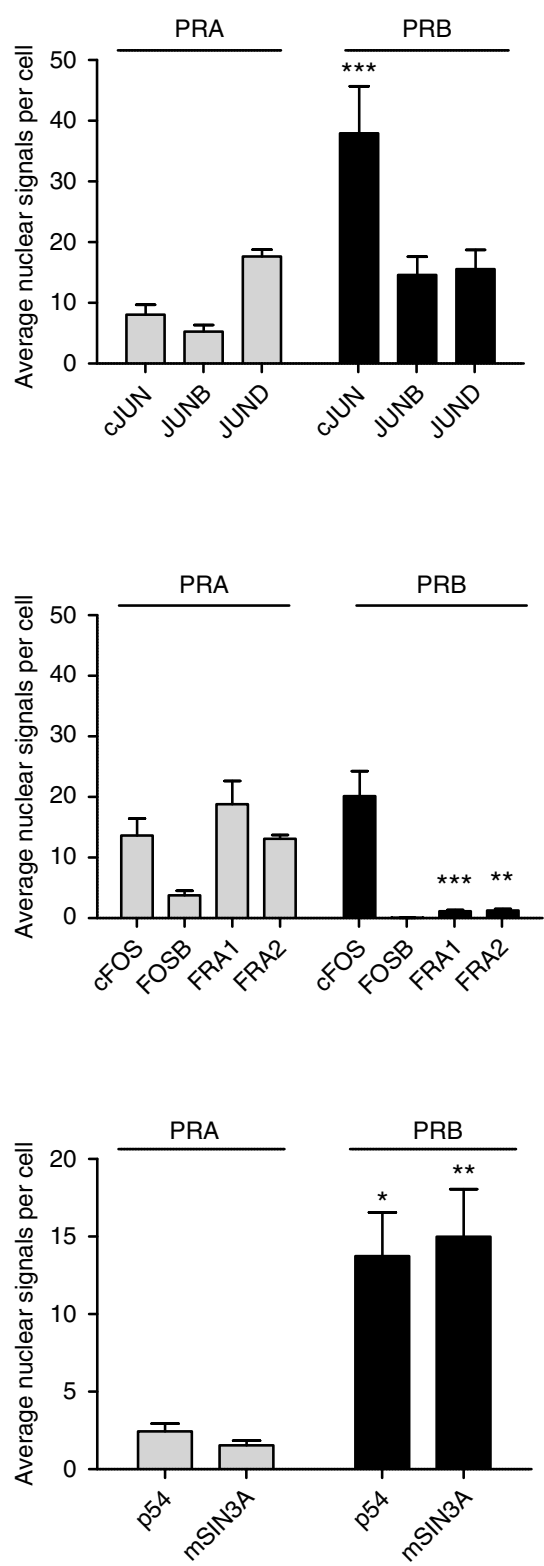

Figure 1 | PRA/PRB show differential interaction with AP-1 transcription factors and transcriptional co-repressor proteins p54 ${ }^{\mathrm{nrb}} / \mathrm{mSIN3A}$. In situ proximity ligation assay (PLA) of PRs with Jun and Fos family members and co-repressor proteins; $\mathrm{p} 54^{\text {nrb }}$ and mSIN3A. PRA and PRB stable transfected hTERT-HM cells were treated with $100 \mathrm{nM}$ progesterone (P4) for $2 \mathrm{~h}$ and then subjected to PLA. (a) Representative pictures and (b) signal analysis (average nuclear signals/cell from three fields), pooled from three independent experiments are shown. Two-way analysis of variance followed by bonferroni post-tests show significant differences between PRA and PRB in the interaction of cJUN $\left(P<0.001\right.$, denoted as $\left.{ }^{\star \star \star}\right)$, Fra1 $(P<0.001)$, Fra2 $\left(P<0.01\right.$, denoted as $\left.{ }^{\star \star}\right)$, p54 $\left(P<0.05\right.$, denoted as $\left.{ }^{\star}\right)$, and mSIN3A $(P<0.01)$. Data represents mean \pm s.d., $n=3$. Scale bar, $20 \mu \mathrm{m}$.

demonstrated that PR is liganded during pregnancy when P4 levels are elevated but are unliganded during labour when peripheral and myometrial P4 levels are reduced (Fig. 3b). In both cases the loss of P4-PR binding was not due to a loss of nuclear $\mathrm{PR}$ as demonstrated by immunofluorescence.

Myometrial P4 level is decreased during labour. Next we examined whether the lack of binding of P4 to PR during human labour was due to reduced myometrial P4 levels despite the abundance of circulating P4. Using immunofluorescence, we found that the nuclear P4 levels were decreased in the labouring human myometrium in comparison with non-labouring myometrium (Fig. 3a). Moreover, we found increased expression of the P4-metabolizing enzyme $20 \alpha$ HSD/AKR1C1 in human myometrium during labour compared with non-labouring tissue (Fig. 3a). These data suggest that metabolism of P4 leads to a local withdrawal of the steroid and consequently uncoupling of PR from its ligand. To verify the reduced tissue levels of P4, we conducted ELISA in myometrial tissue lysates from non- 

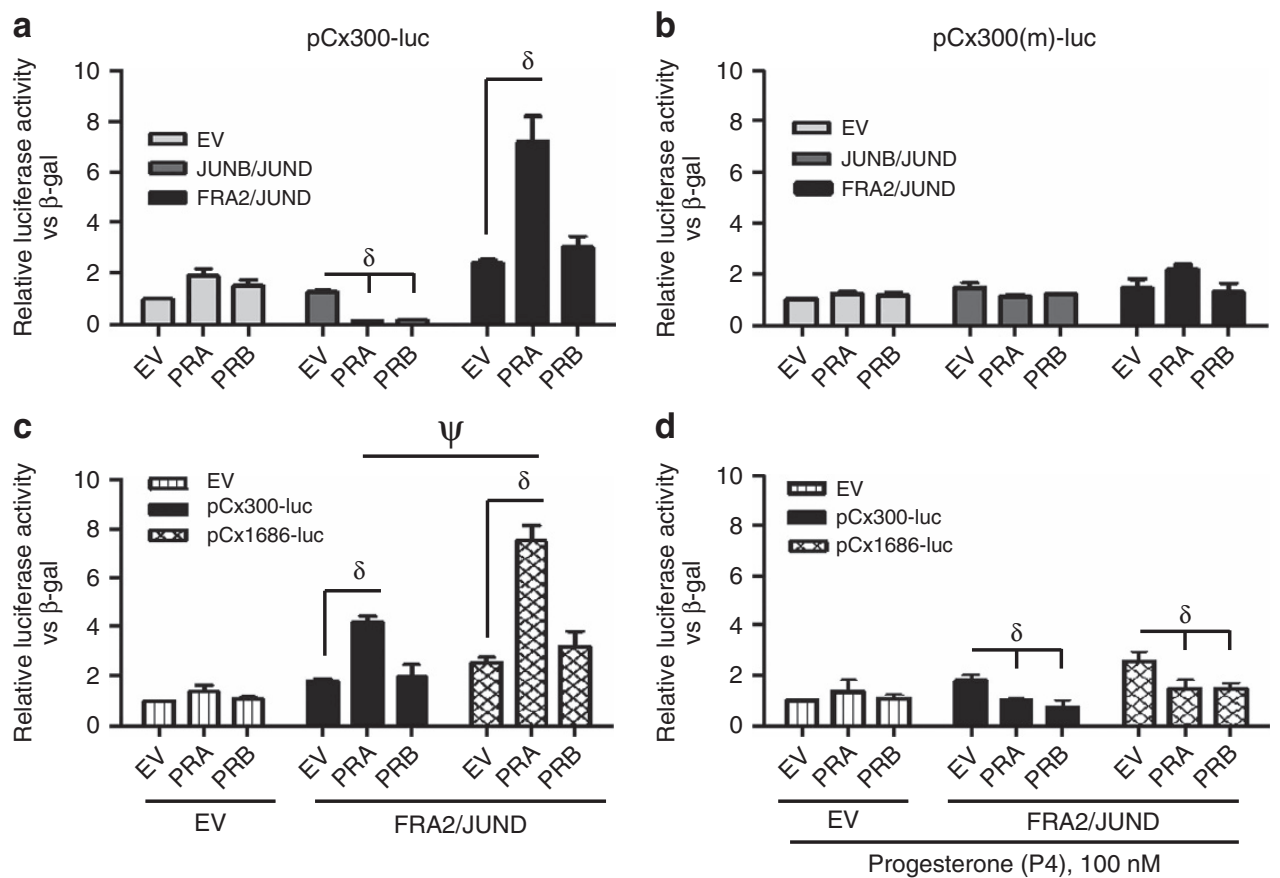

Figure 2 | Unliganded PRA activates Cx43 transcription in the presence of Fos/Jun heterodimer. SHM cells were transiently transfected with pCx300luc, pRSVbgal, PRA or PRB, and (1:1) combination of Jun/Jun or Fos/Jun expression vectors for $48 \mathrm{~h}$. The relative luciferase to $\beta$-galactosidase activity is represented as the fold induction over cells transfected with the empty vector control (EV of the EV group). (a) Luciferase promoter activity assay: PRs suppress $\mathrm{C} \times 43$ transcription in the presence of Jun/Jun dimers but induces it in the presence of Fos/Jun heterodimer (FRA2/JUND). (b) Mutation of AP-1 sequence abolishes PRA mediated transactivation of $\mathrm{C} \times 43$ promoter. (c) Increasing the number of AP-1 sites enhances the transactivation of $\mathrm{C} \times 43$ promoter by PRA in the presence of Fos/Jun heterodimer. (d) Addition of progesterone (P4) suppressed Cx43 promoter activity by PRs in the presence of heterodimer. $\delta$ represents significant difference $(P<0.05)$ with respect to $E V$ control of that group and $\psi$ represents significant difference $(P<0.05)$ in the PRA of the respective groups as determined by two-way analysis of variance. Data represents mean \pm s.d., $n=4$ independent experiments.

labouring and labouring women $(n=11$ per group). We compared cytoplasmic and nuclear P4 levels in human myometrium and found that P4 concentration was three- to fourfold higher in the cytoplasm than in the nucleus. There was no significant difference in the cytoplasmic P4 concentration between labouring and non-labouring human myometrium; however, a significant decrease $(P<0.006)$ in the nuclear $\mathrm{P} 4$ levels was detected during term labour (Fig. 4).

Differential localization of liganded and unliganded PRs. To validate the in vivo $\mathrm{P} 4-\mathrm{PR}$ interactions, we used human myometrial cells (hTERT-HM ${ }^{\mathrm{A} / \mathrm{B}}$ ) expressing doxycycline (DOX)inducible PRA and RheoSwitch ligand (RSL)-inducible $\mathrm{PRB}^{32}$ (expression validation shown in Supplementary Fig. 6), and performed in situ PLA in the presence or the absence of P4. These in vitro assays revealed a distinct intracellular localization of P4-liganded PRA and PRB: P4-liganded PRA was found in the cytoplasm but was absent in the nuclei (Fig. 5b), whereas P4-liganded PRB was strictly localized in the nucleus (Fig. 5h). In the unliganded state, PRA was primarily localized in the nucleus (Fig. 5c), whereas PRB was found to be exclusively cytoplasmic in localization (Fig. 5i,k). Furthermore, western blot of cytoplasmic and nuclear fractions showed that $\mathrm{P} 4$ treatment resulted in the translocation of PRB to the nucleus, while PRA remained predominantly within the cytoplasm (Fig. 5m, Supplementary Fig. 7). Since PRs are unliganded during labour (Fig. 3a,b), we suggest that PRA accounts for the majority of unliganded nuclear PRs in the labouring myometrium.

PR is unliganded during preterm labour. Finally, given the importance of preterm labour as a major driver of perinatal mortality and morbidity in newborns, we examined whether the mechanisms responsible for $\mathrm{P} 4$ withdrawal during preterm labour were similar to that of term labour. Myometrial biopsies were collected at emergency $\mathrm{C}$-section from women in active preterm labour and from a pregnancy undergoing second trimester C-section not-in-labour. Importantly, PLA analysis revealed myometrial PRs were unliganded during preterm labour (Fig. 6), whereas the PRs in the preterm myometrium not-in-labour were liganded. Moreover, while there was abundant myometrial nuclear PRs during preterm labour, there was a reduction in intracellular P4 as detected by immunofluorescence (Fig. 6). These data suggest that the reduced intracellular P4, the dissociation of PRs from P4 and the presence of unliganded PRs within the nucleus represents a common element of both term and preterm human labour.

\section{Discussion}

In this study we have identified a mechanism by which the pregnancy-maintaining action of $\mathrm{P} 4$ is withdrawn in association with the onset of human labour, even in the presence of elevated circulating levels of this hormone. We show that at the time of human labour PR (predominantly PRA) dissociates from P4 and, therefore, becomes unliganded. We also show that the unliganded PRA localizes to the nucleus where, rather than acting as a repressor of $C x 43$ gene transcription, it paradoxically activates transcription of this key labour gene, through mechanisms involving PRA interactions with AP-1 heterodimers. Thus, our data provide a mechanistic explanation of Csapo's 'functional progesterone withdrawal' theory some 50 years after he postulated how labour could occur in women despite the maintenance of elevated levels of circulating progesterone. 
a
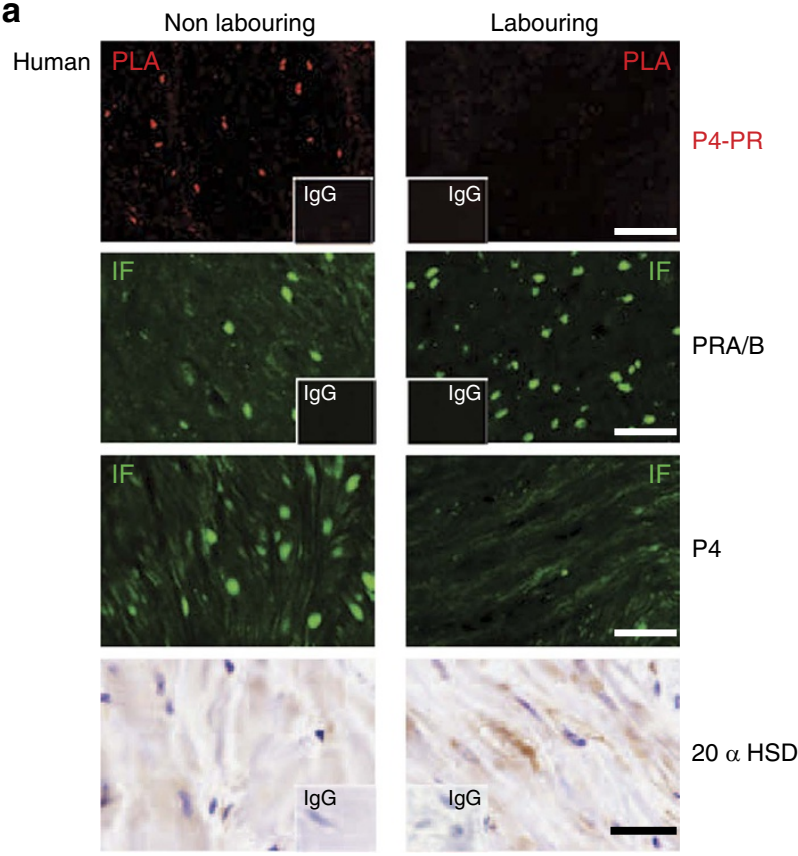

b
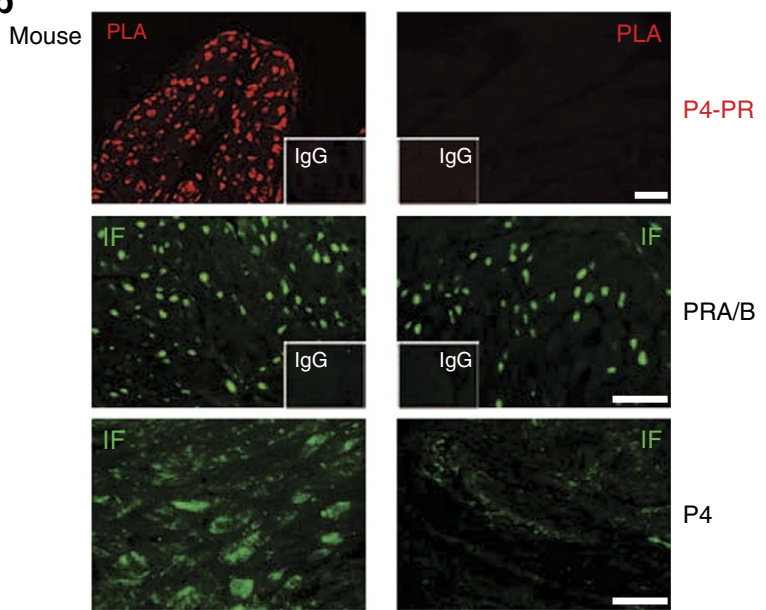

Figure 3 | Myometrial PRs are unliganded during labor. (a) Term human myometrium from non-labouring and labouring state ( $n=4$ per group). Representative pictures of: in situ Proximity Ligation Assay (PLA) between PR and P4 where red signal represents P4-liganded PRs, and immunofluorescence (IF) with green signal represents total PRs (PRA/B) and P4, and immunohistochemistry (IHC) for 20 $\alpha$ HSD. (b) Mouse myometrium from non- labouring and labouring state ( $n=4$ per group). IgG controls shown as embedded picture. Scale bar, $50 \mu \mathrm{m}$.

Our data indicate marked differences by which the two PR isoforms, PRA and PRB modulate gene transcription in the pregnant myometrium. During pregnancy under the influence of P4, PRB forms a complex with transcriptional repressors $\mathrm{p} 54^{\mathrm{nrb}} /$ $\mathrm{mSIN} 3 \mathrm{~A} / \mathrm{HDAC}$ and inhibits expression of Cx43 (ref. 28). During human labour, we have reported that there is an increase in the ratio of PRA/PRB and suggested that PRA acts to antagonize the repressive functions of $\mathrm{PRB}$ on labour gene expression ${ }^{6}$. Here we suggest that it is the unliganded status of PRA that determines its properties as a transcriptional activator. Only in its unliganded state can PRA drives Cx43 transcription. Studies in human breast cancer support this assertion in that the PRB isoform is transcriptionally active when bound to P4, while PRA is transcriptionally active in its unliganded state ${ }^{33}$.

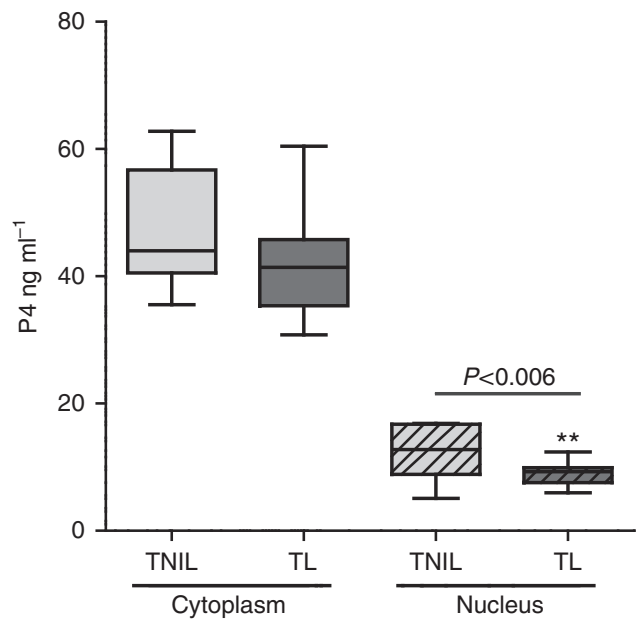

Figure 4 | Nuclear P4 levels are decreased in the labouring myometrium. Cytoplasmic and Nuclear tissue lysates were prepared from non-labouring and labouring human myometrium $(n=11$ each). Lysates were subjected to P4 ELISA using DRG Progesterone Enzyme Immunoassay Kit (DRG, Germany). Statistical analysis was performed using $t$-test for equality of variance. Significance was determined at ${ }^{\star} P<0.01$. Data represents mean \pm s.d.

The liganded status of the PR isoforms also has a marked effect on their cellular localization. Classically, unliganded steroid receptors are found in the cytoplasm and are transcriptionally inactive, whereas on binding to their ligand the receptors translocate to the nucleus where they regulate gene transcription. Here we show that while PRB follows this canonical model, PRA exhibits a completely different pattern of cellular localization. In the presence of P4, PRA is cytoplasmic but in the unliganded state it is found predominantly in the nucleus where it induces Cx43 transcription. Differential subcellular distribution of PRA and PRB has been previously described for endometrial and breast cancer cells ${ }^{34,35}$. In those studies, in the absence of P4, PRB was localized within the cytoplasm and nucleus, but was primarily nuclear in the presence of P4. In contrast, PRA was predominantly nuclear even in the absence of P4. Unfortunately, those previous studies were not able to discriminate between $\mathrm{P} 4$-liganded versus unliganded status of the isoforms.

We show that the ability of PR isoforms to modulate transcription of $\mathrm{Cx} 43$ is also dependent on the composition of AP-1 dimers on the $\mathrm{Cx} 43$ promoter. JUN/JUN homodimers favour the binding of $\mathrm{PRB}$ and the interaction of $\mathrm{PRB}$ with the transcriptional repressor complex, $\mathrm{p} 54^{\mathrm{nrb}} / \mathrm{mSIN} 3 \mathrm{~A} / \mathrm{HDAC}$. In contrast, FRA2/JUND heterodimers have reduced affinity for PRB, but instead strongly interact with PRA and induce Cx43 transcriptional activity. Nuclear FRA2 and JUND expression is increased (Supplementary Fig. 2) and, therefore, we suggest that during pregnancy a complex including liganded PRB, JUN/JUN and $\mathrm{p} 54^{\mathrm{nrb}} / \mathrm{mSIN} 3 \mathrm{~A} / \mathrm{HDAC}$ suppresses transcription of $\mathrm{Cx} 43$, while during labour the unliganded PRA-FRA2/JUND complex is responsible for the increased transcription of this labour gene. Importantly, this mechanism may be applicable to other labourassociated genes, including PTGS2, OXTR, OXN, PTGDS and NFKB2 and several pro-inflammatory cytokines, chemokines and extracellular matrix proteins, which are also known to be regulated by AP-1 transcription factors.

The mechanism by which PRA becomes unliganded at the time of labour remains to be determined. However, Runnebaum and Zander ${ }^{36}$ reported that myometrial $\mathrm{P} 4$ levels were reduced and levels of its metabolite, $20 \alpha$-dihydroprogesterone were increased in the human myometrium during labour, while Williams et al. ${ }^{37}$ 

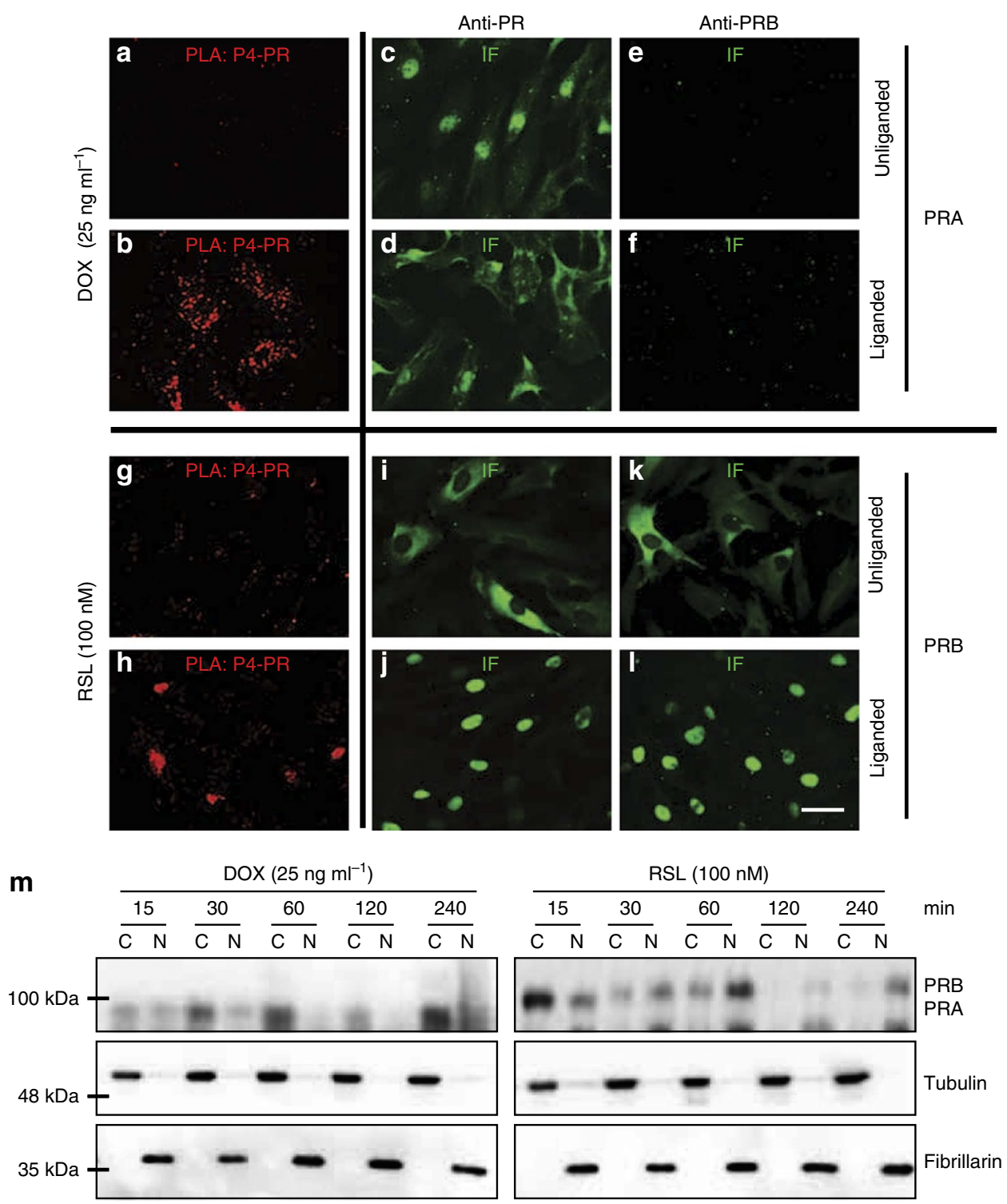

Figure 5 | Liganded and unliganded PRA and PRB isoforms have differential cellular distribution. Representative pictures of: in situ Proximity Ligation Assay (PLA) and immunofluorescence (IF) for total PRs and PRB in human myometrial cells with inducible PRA (a-f) and PRB (g-I) expression system. Cells were induced for $24 \mathrm{~h}$ with Dox $\left(25 \mathrm{ng} \mathrm{ml}^{-1}\right)$ for PRA and with RSL (100 nM) for PRB expression and then treated with vehicle or P4 (100 $\left.\mathrm{nM}\right)$ for $2 \mathrm{~h}$ before subjecting to fixation and PLA analysis. Scale bar, $40 \mu \mathrm{m}$. (m) Cytoplasmic (C) and nuclear (N) fractionation analysis of PRA and PRB following stimulation with P4 for 15-240 min demonstrates gradual accumulation of PRB in the nucleus and PRA in the cytoplasm. Representative western blots and fractionation controls (Tubulin and Fibrillarin) are shown.

recently reported that labour is associated with increased expression of the P4 metabolizing enzyme 20 $\alpha$ HSD. Our finding that the presence of the unliganded PRA occurs in association with increased expression of $20 \alpha$ HSD and reduced nuclear levels of P4 suggests that intracellular metabolism of P4 may lead to its dissociation from its receptor leading to the downstream events that result in labour.

In summary, our data represent a paradigm shift in our understanding of the mechanisms by which $\mathrm{P} 4$ (through its receptors) controls myometrial contractility during pregnancy and labour. We show that while liganded nuclear PRB can suppress the expression of Cx43, unliganded PRA paradoxically translocates to the nucleus where it acts as a transcriptional activator of this labour gene (Fig. 7). We provide evidence that metabolism of P4 may underlie the reduced nuclear levels of this hormone and its dissociation from its receptor. There has been considerable interest recently in the use of $\mathrm{P} 4$ to prevent preterm labour $^{38-40}$, though clinical trials suggest that this therapy is only successful in a subset of patients. Our study raises the possibility that a progestogen that is not subject to metabolism by $20 \alpha$ HSD might represent a more effective therapeutic approach to the prevention of the mortality and morbidity associated with prematurity.

\section{Methods}

Cell lines and cell culture. Human telomerase immortalized myometrial cells (hTERT-HM) originally developed by Dr Jennifer Condon ${ }^{30}$, were engineered to stably express Flag-PRA or Flag-PRB ${ }^{29}$. Briefly, lentiviral mock vector (backbone: pFUGWBW), Flag-PRA and Flag-PRB expression constructs were transfected into 293T cells along with virapower packaging mix ( $9 \mu \mathrm{g}$, Invitrogen) for $48 \mathrm{~h}$.

Lentiviral particles harvested from the conditioned medium of the transfected cells were used to infect hTERT-HM cells. Stable cell lines including hTERT(mock), hTERT(PRA) and hTERT(PRB) were generated after 3 weeks (W) of selection with blasticidin $\left(10 \mathrm{ng} \mathrm{\mu l}^{-1}\right)$. hTERT-HM ${ }^{\mathrm{A} / \mathrm{B}}$ cell line, with DOX-inducible PRA and RSL-inducible PRB expression, were previously described ${ }^{32}$. Briefly, an hTERT- 
HM subline was generated by stable transfection of linearized Tet-ON-advanced plasmid and neomycin resistance expression plasmid. Neomycin resistant subline was transfected with linearized pTetON-advanced plasmid with an insert of PRA open reading frame along with linearized DNA for hygromycin resistance. The subline with hygromycin resistance was tested for DOX-mediated induction of PRA expression and further transfected with a RheoSwitch plasmid carrying open reading frame for $\mathrm{PRB}$ along with the linearized DNA for basticidin resistance gene and finally the blasticidin resistant subline (hTERT-HM ${ }^{\mathrm{A} / \mathrm{B}}$ ) was screened for the
Preterm non labouring
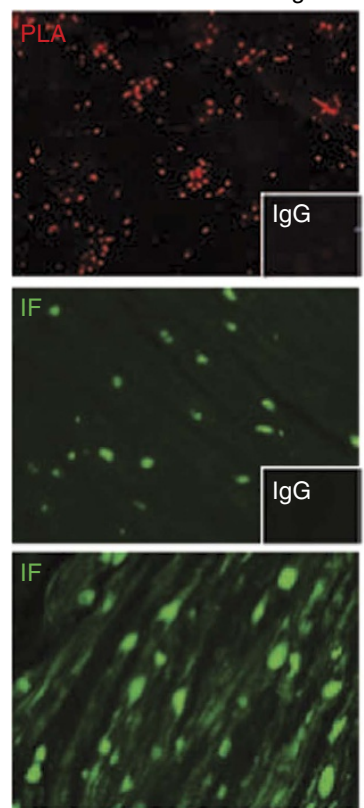

Preterm labour
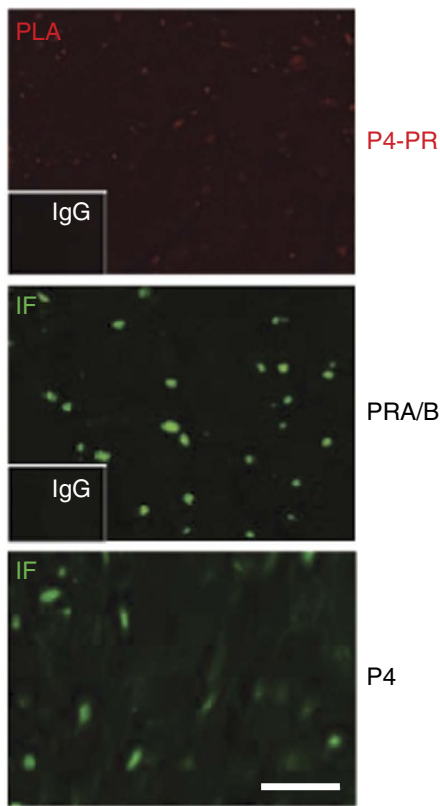

Figure 6 | Myometrial PRs are unliganded during preterm labour. Human myometrium from preterm control (non-labouring) and preterm labour. Representative pictures $(n=3)$ of: in situ Proximity Ligation Assay (PLA) between PR and P4 where red signal represents P4-liganded PRs, and immunofluorescence (IF) with green signal represents total PRs (PRA/B) and P4. Scale bar, $40 \mu \mathrm{m}$.
DOX induciblity of PRA and RSL inducibility of PRB expression. SHM cells were maintained in DMEM supplemented with $10 \% \mathrm{FBS}, 100 \mathrm{IU} \mathrm{ml}^{-1}$ penicillin and $100 \mu \mathrm{g} \mathrm{ml}^{-1}$ streptomycin. Media for hTERT-HM stable cells were further supplemented with the resistance antibiotic; blasticidin $5 \mu \mathrm{g} \mathrm{ml}^{-1}$ and that of hTERT-HM ${ }^{\mathrm{A} / \mathrm{B}}$ was supplemented with $100 \mu \mathrm{g} \mathrm{ml}^{-1}$ of Geneticin, $1 \mu \mathrm{g} \mathrm{ml}^{-1}$ of Hygromycin B and $5 \mu \mathrm{g} \mathrm{ml}^{-1}$ Blasticidin. All the reagents for cell culture were purchased from Invitrogen Canada, Inc. All the cell lines used in this study were mycoplasma free as assessed by DAPI-DNA staining.

In situ proximity ligation assays. Protein-protein interactions were detected using the Duolink II in situ Proximity Ligation Assays (PLA) Detection Kit (Olink Bioscience, Sweden). Briefly, the hTERT-HM PRA and PRB stable cells were cultured on 16-well chamber slides (Lab Tek, Fisher, CA), serum starved for $24 \mathrm{~h}$ in serum-free medium and then stimulated with Progesterone $(\mathrm{P} 4,100 \mathrm{nM})$ for $2 \mathrm{~h}$. Cells were then washed with cold PBS and fixed with cold methanol: acetone (1:1) for $3 \mathrm{~min}$. After washing, cells were permeabilized with $0.2 \%$ Triton X-100 for $5 \mathrm{~min}$, blocked with Olink blocking solution for $1 \mathrm{~h}$ and incubated with primary antibodies for overnight at $4{ }^{\circ} \mathrm{C}$. Selection of primary antibodies from different species sources was carefully performed and chosen antibodies presented negligible background. Antibodies from the same secondary source were preconjugated with probes to avoid cross reactions. Hybridization with PLA probes (plus and minus), ligation and amplification of conjugants was performed as per manufacturer's instructions. Stringent negative controls were included in all experiments. Slides were mounted with DAPI containing anti-fade mounting medium (Olink, Bioscience) and pictures were taken at constant exposure and $\times 200$ magnification by Leica DM IL LED-Inverted fluorescence microscope with micropublisher 5.0 RTV Q imaging system. Images were analysed by Duolink Image analysis software according to the instructions.

Collection of murine gestational myometrial tissues. Hsd:ICR (CD-1) outbred mice were mated and vaginal plug detection day was considered day 0.5 of pregnancy. Animals were killed and myometrial samples were collected on gestational day (GD) 15 (non-labouring) and during term labour (GD19). Tissue was collected at 10:00 hours ( $n=3$ or more/GD) with the exception of GD19 sample that was collected during active labour as was reported previously ${ }^{11}$. Uterine horns were bisected longitudinally and dissected away from pups and placentas in ice-cold PBS. The decidua basalis and decidua parietalis were removed from the myometrial tissue by cutting/mechanical scraping on ice, respectively, as described earlier ${ }^{41}$. The myometrial tissue was frozen in liquid nitrogen and stored at $-80^{\circ} \mathrm{C}$. All mice were housed under specific pathogen-free conditions at the Toronto Centre for Phenogenomics and all animal experiments were approved by the Toronto Centre for Phenogenomics Animal Care Committee.

Collection of human non-labouring and labouring myometrial tissues. The study design was approved by the Institutional Research Ethics Board of Mount Sinai Hospital, Toronto. Healthy pregnant women with a singleton pregnancy

\begin{abstract}
P4 Progesterone
p54 Transcriptional co-repressor

mSIN3A p54 binding partner and co-repressor

HDAC Histone deacetylase
\end{abstract}

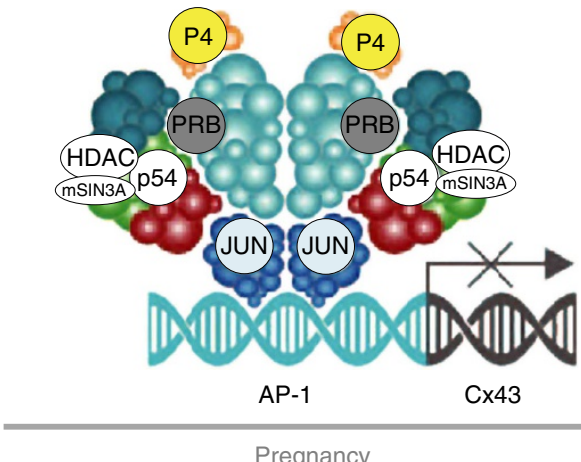

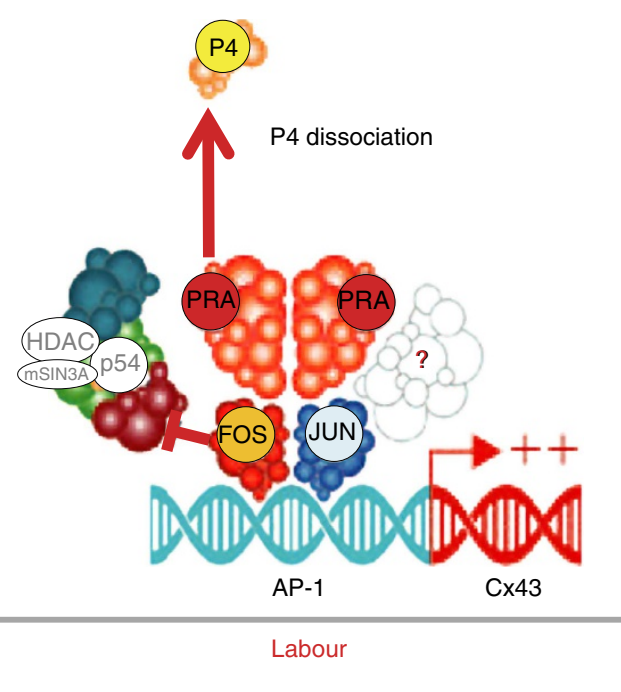

Figure 7 | Hypothetical model of Cx43 regulation during pregnancy and term (or preterm) labour. During gestation Jun/Jun dimer complex dominates and interacts with P4-liganded PRs/p54 nrb/mSIN3A-HDAC transcriptional repressor complex which is recruited to the Cx43 promoter and represses Cx43 transcription. Before the onset of term labour or in the incidence of preterm labour, P4-PR dissociation and simultaneous upregulation of PRA and Fos (FRA2) results in PRA-Fos/Jun heterodimer complex formation (which does not allow incorporation of co-repressor proteins but may facilitate binding of unknown co-activators marked as '?'), transcriptional activation of $\mathrm{C} \times 43$ and initiation of labour. 
undergoing elective caesarean delivery at term (gestational age $\geq 37 \mathrm{~W}$ ) were recruited for 'non-labouring' myometrial tissue collection $(n=8)$. Caesarean delivery of 'labouring' $(n=6)$ patients was performed after the onset of labour (regular uterine contractions every $10 \mathrm{~min}$, cervical dilatation $>3 \mathrm{~cm}$ ). Similarly, tissue biopsies were collected from women undergoing emergency C-section in active preterm labour $(n=3$, one case of chorioamnionitis (28W) and two idiopathic preterms $(29+7 \mathrm{~W}, 34 \mathrm{~W})$ and from pregnancies undergoing second trimester C-section not-in-labour $(n=3,32 \mathrm{~W}, 31+6 \mathrm{~W}, 29+4 \mathrm{~W})$. A written consent to participate in the study was obtained from each patient. The myometrial biopsy sample $\left(\sim 1 \mathrm{~cm}^{3}\right)$ was excised from the upper margin of an incision made in the lower uterine segment after delivery of the fetus. The biopsy was washed in icecold PBS and immediately placed in 10\% neutral-buffered formalin (Harleco, Baltimore, MD) or 4\% paraformaldehyde (Electron Microscopy Sciences, Hartfield, PA) for fixation. Samples were fixed for $24 \mathrm{~h}$ at $4{ }^{\circ} \mathrm{C}$.

\section{Quantification of P4 levels in myometrial tissue by ELISA. P4 ELISA was} conducted on the cytoplasmic and nuclear tissue lysates were prepared from non-labouring and labouring human myometrium ( $n=11$ each), using DRG Progesterone Enzyme Immunoassay Kit, DRG, Germany) as per manufacturer's instructions. Statistical analysis was performed using $t$-test for equality of variance.

Immunohistochemical staining of human myometrial tissue. Immunohistochemistry and immunofluorescence experiments were performed using standard procedures as previously described ${ }^{41,42}$. Antibodies used in immunohistochemistry and immunofluorescence are listed in Supplementary Table 1.

Protein extraction and immunoblotting. Total cell lysates were prepared in lysis buffer $(0.08 \mathrm{M}$ Tris/ $\mathrm{HCl}$ (pH 6.8), 2\% SDS, $10 \%$ Glycerol) with freshly added protease and phosphatase inhibitor cocktail (Thermo Fisher Scientific, Inc.). Cytoplasmic and nuclear lysates from tissues were prepared with NE-PER, Nuclear and Cytoplasmic Extraction kit (Pierce, USA). Equal amount of protein was separated by SDS-PAGE and transferred to a polyvinylidene difluoride membrane (Trans-blot Turbo Midi PVDF, Bio-Rad) using Turbo Trans-Blot system (BioRad). After blocking for an hour with 5\% milk in TBS-T, the membranes were incubated with primary antibody at $4{ }^{\circ} \mathrm{C}$ for overnight. The membranes were subsequently probed with horseradish peroxidase-conjugated secondary antibody at room temperature for $1 \mathrm{~h}$. Signals were detected using Luminata HRP-substrate (Millipore) and imaging was performed with VersaDoc imaging system (Bio-Rad). Antibodies used for immunoblotting are listed in Supplementary Table 1. $\alpha$-tubulin (Sigma-Aldrich, USA), was used as loading control for whole cell lysate and fractionation control for cytosolic proteins, whereas Fibrillarin (Cell Signalling, USA), were used as fractionation controls for nuclear proteins. Secondary antibodies for rabbit, mouse, and goat were purchased from Amersham (dilution range; 1:5,000-10,000) and normal IgGs from Santa Cruz. Densitometric analysis was performed using Image Lab system (Bio-Rad, USA). All uncropped western blots can be found in Supplementary Fig. 7 .

Transient transfection. Transient transfection was performed using jetPRIME (Polyplus) following manufacturer's suggested procedures. The PR expression construct pSG5-PRA and pSG5-PRB, Jun and Fos constructs (pcDNA3.1-cJun, pcDNA3.1- JunB, pcDNA3.1- JunD, pcDNA3.1- cFos, pcDNA3.1- FosB, pcDNA3.1- Fra1 and pcDNA3.1- Fra2), and Cx43 Luciferase promoter constructs (pCx1686-luc, pCx300-luc, and pCx300(m)-luc) were described earlier ${ }^{26,43}$.

Co-Immunoprecipitation. Cells were washed twice with ice-cold PBS and lysed with buffer containing $25 \mathrm{mMTris}-\mathrm{HCl}$ (pH 7.4), $150 \mathrm{mM} \mathrm{NaCl}, 1 \mathrm{mM}$ EDTA, $1 \%$ NP-40, 5\% Glycerol and freshly added protease \& phosphatase inhibitor cocktail (Thermo Fisher Scientific Inc. 1:100). Protein samples $(500-1000 \mu \mathrm{g})$ were pre-cleared with washed protein A or G conjugated agarose beads for $2 \mathrm{~h}$ at $4{ }^{\circ} \mathrm{C}$ and the supernatant was incubated with $2-4 \mu \mathrm{g}$ antibody/IgG control, overnight at $4{ }^{\circ} \mathrm{C}$ and then applied to pre-washed Agrose beads $2 \mathrm{~h}$ at $4{ }^{\circ} \mathrm{C}$. Agarose beads were then washed three times with Lysis buffer and immunoprecipitates were collected by boiling beads in $40-50 \mu$ l of $2 \times$ Laemmli buffer for 10 min. Finally, the supernatant and $10 \%$ input was subjected to SDS-PAGE and western blot analysis.

Luciferase reporter assay. SHM cells were transfected with PRA or PRB, different combinations of AP-1 expression vectors (1:1), luciferase reporter for $\mathrm{Cx} 43$ promoter (pCx300-luc, $\mathrm{pCx} 300(\mathrm{~m})$-luc or $\mathrm{pCx} 1680$-luc), and pRSV $\beta$ gal vector (containing Escherichia coli lacZ gene under the Rous sarcoma virus promoter). Cells were recovered $5 \mathrm{~h}$ after transfection and treated with $\mathrm{P} 4$ or its vehicle for $24 \mathrm{~h}$ and then collected in passive lysis buffer (Promega, Madison, WI, USA). Luciferase activity was determined using luciferin reagent from Promega. Transfection efficiency was normalized with $\beta$-galactosidase activity. All the experiments were performed in triplicates and repeated at least thrice.

Statistical analysis. Differences among several groups were determined by oneway analysis of variance, followed by Dunnets Multiple comparison test using
Prism software (GraphPad Prism; San Diego, CA). Two-way analysis of variance and Bonferroni post-tests were used to compare different variables.

\section{References}

1. Corner, G. W. \& Allen, W. M. Physiology of the corpus luteum. II. Production of a Special uterine reaction (progestational proliferation) by extracts of the corpus luteum. Am. J. Physiol. 88, 326 (1929).

2. Csapo, A. Progesterone block. Am. J. Anat. 98, 273-291 (1956).

3. Csapo, A. I. \& Pinto-Dantas, C. A. The effect of progesterone on the human uterus. Proc. Natl Acad. Sci. USA 54, 1069-1076 (1965).

4. Evans, J. J., Sin, I. L., Duff, G. B. \& Frampton, C. M. Estrogen-induced transcortin increase and progesterone and cortisol interactions: implications from pregnancy studies. Ann. Clin. Lab. Sci. 17, 101-105 (1987).

5. Mitchell, B. F. \& Wong, S. Changes in 17 beta, 20 alpha-hydroxysteroid dehydrogenase activity supporting an increase in the estrogen/progesterone ratio of human fetal membranes at parturition. Am. J. Obstet. Gynecol. 168, 1377-1385 (1993).

6. Merlino, A. A. et al. Nuclear progesterone receptors in the human pregnancy myometrium: evidence that parturition involves functional progesterone withdrawal mediated by increased expression of progesterone receptor-A. J. Clin. Endocrinol. Metab. 92, 1927-1933 (2007).

7. Condon, J. C., Jeyasuria, P., Faust, J. M., Wilson, J. W. \& Mendelson, C. R. A decline in the levels of progesterone receptor coactivators in the pregnant uterus at term may antagonize progesterone receptor function and contribute to the initiation of parturition. Proc. Natl Acad. Sci. USA 100, 9518-9523 (2003).

8. Haluska, G. J., West, N. B., Novy, M. J. \& Brenner, R. M. Uterine estrogen receptors are increased by RU486 in late pregnant rhesus macaques but not after spontaneous labor. J. Clin. Endocrinol. Metab. 70, 181-186 (1990).

9. Allport, V. C. et al. Human labor is associated with nuclear factor-kappaB activity which mediates cyclo-oxygenase- 2 expression and is involved with the 'functional progesterone withdrawal'. Mol. Hum. Reprod. 7, 581-586 (2001).

10. Dudley, D. J., Branch, D. W., Edwin, S. S. \& Mitchell, M. D. Induction of preterm birth in mice by RU486. Biol. Reprod. 55, 992-995 (1996).

11. Shynlova, O., Nedd-Roderique, T., Li, Y., Dorogin, A. \& Lye, S. J. Myometrial immune cells contribute to term parturition, preterm labor and post-partum involution in mice. J. Cell Mol. Med. 17, 90-102 (2013).

12. Maria, B., Stampf, F., Goepp, A. \& Ulmann, A. Termination of early pregnancy by a single dose of mifepristone (RU 486), a progesterone antagonist. Eur. J. Obstet. Gynecol. Reprod. Biol. 28, 249-255 (1988).

13. Kokubu, K., Hondo, E., Sakaguchi, N., Sagara, E. \& Kiso, Y. Differentiation and elimination of uterine natural killer cells in delayed implantation and parturition mice. J. Reprod. Dev. 51, 773-776 (2005).

14. Kastner, P. et al. Two distinct estrogen-regulated promoters generate transcripts encoding the two functionally different human progesterone receptor forms A and B. EMBO J. 9, 1603-1614 (1990).

15. Sartorius, C. A. et al. A third transactivation function (AF3) of human progesterone receptors located in the unique $\mathrm{N}$-terminal segment of the B-isoform. Mol. Endocrinol. 8, 1347-1360 (1994).

16. Vegeto, E. et al. Human progesterone receptor A form is a cell- and promoterspecific repressor of human progesterone receptor B function. Mol. Endocrinol. 7, 1244-1255 (1993).

17. Dong, X., Challis, J. R. \& Lye, S. J. Intramolecular interactions between the AF3 domain and the C-terminus of the human progesterone receptor are mediated through two LXXLL motifs. J. Mol. Endocrinol. 32, 843-857 (2004).

18. Orsino, A., Taylor, C. V. \& Lye, S. J. Connexin- 26 and connexin- 43 are differentially expressed and regulated in the rat myometrium throughout late pregnancy and with the onset of labor. Endocrinology 137, 1545-1553 (1996).

19. Sakai, N., Tabb, T. \& Garfield, R. E. Modulation of cell-to-cell coupling between myometrial cells of the human uterus during pregnancy. Am. J. Obstet. Gynecol. 167, 472-480 (1992).

20. Balducci, J. et al. Gap junction formation in human myometrium: a key to preterm labour? Am. J. Obstet. Gynecol. 168, 1609-1615 (1993).

21. Cook, J. L., Zaragoza, D. B., Sung, D. H. \& Olson, D. M. Expression of myometrial activation and stimulation genes in a mouse model of preterm labor: myometrial activation, stimulation, and preterm labor. Endocrinology 141, 1718-1728 (2000).

22. Tong, D. et al. A dominant loss-of-function GJA1 (Cx43) mutant impairs parturition in the mouse. Biol. Reprod. 80, 1099-1106 (2009).

23. Doring, B. et al. Ablation of connexin 43 in uterine smooth muscle cells of the mouse causes delayed parturition. J. Cell Sci. 119, 1715-1722 (2006).

24. Halazonetis, T. D., Georgopoulos, K., Greenberg, M. E. \& Leder, P. c-Jun dimerizes with itself and with c-Fos, forming complexes of different DNA binding affinities. Cell 55, 917-924 (1988). 
25. Geimonen, E. et al. Activation of protein kinase $C$ in human uterine smooth muscle induces connexin-43 gene transcription through an AP-1 site in the promoter sequence. J. Biol. Chem. 271, 23667-23674 (1996).

26. Mitchell, J. A. \& Lye, S. J. Differential activation of the connexin 43 promoter by dimers of activator protein-1 transcription factors in myometrial cells. Endocrinology 146, 2048-2054 (2005).

27. Mitchell, J. A. \& Lye, S. J. Regulation of connexin43 expression by c-fos and c-jun in myometrial cells. Cell Commun. Adhes. 8, 299-302 (2001).

28. Dong, X. et al. p54nrb is a transcriptional corepressor of the progesterone receptor that modulates transcription of the labor-associated gene, connexin 43 (Gja1). Mol. Endocrinol. 23, 1147-1160 (2009).

29. Xie, N. et al. Expression and function of myometrial PSF suggest a role in progesterone withdrawal and the initiation of labor. Mol. Endocrinol. 26, 1370-1379 (2012)

30. Condon, J. et al. Telomerase immortalization of human myometrial cells. Biol. Reprod. 67, 506-514 (2002).

31. Chen, Z. Q. et al. Identification of two regulatory elements within the promoter region of the mouse connexin 43 gene. J. Biol. Chem. 270, 3863-3868 (1995).

32. Tan, H., Yi, L., Rote, N. S., Hurd, W. W. \& Mesiano, S. Progesterone receptor-A and $-\mathrm{B}$ have opposite effects on proinflammatory gene expression in human myometrial cells: implications for progesterone actions in human pregnancy and parturition. J. Clin. Endocrinol. Metab. 97, E719-E730 (2012).

33. Jacobsen, B. M., Schittone, S. A., Richer, J. K. \& Horwitz, K. B. Progesteroneindependent effects of human progesterone receptors (PRs) in estrogen receptor-positive breast cancer: $\mathrm{PR}$ isoform-specific gene regulation and tumor biology. Mol. Endocrinol. 19, 574-587 (2005).

34. Lim, C. S. et al. Differential localization and activity of the A- and B-forms of the human progesterone receptor using green fluorescent protein chimeras. Mol. Endocrinol. 13, 366-375 (1999).

35. Leslie, K. K. et al. Progesterone receptor isoform identification and subcellular localization in endometrial cancer. Gynecol. Oncol. 96, 32-41 (2005).

36. Runnebaum, B. \& Zander, J. Progesterone and 20 alpha-dihydroprogesterone in human myometrium during pregnancy. Acta Endocrinol. Suppl. 150, 3-45 (1971).

37. Williams, K. C., Renthal, N. E., Condon, J. C., Gerard, R. D. \& Mendelson, C. R. MicroRNA-200a serves a key role in the decline of progesterone receptor function leading to term and preterm labor. Proc. Natl Acad. Sci. USA 109, 7529-7534 (2012).

38. Norwitz, E. R. \& Caughey, A. B. Progesterone supplementation and the prevention of preterm birth. Rev. Obstet. Gynecol. 4, 60-72 (2011).

39. Romero, R., Yeo, L., Chaemsaithong, P., Chaiworapongsa, T. \& Hassan, S. S. Progesterone to prevent spontaneous preterm birth. Semin. Fetal Neonatal. Med. 19, 15-26 (2014).

40. Farine, D. et al. The use of progesterone for prevention of preterm birth. J. Obstet. Gynaecol. Can. 30, 67-77 (2008).
41. Shynlova, O., Tsui, P., Dorogin, A., Chow, M. \& Lye, S. J. Expression and localization of alpha-smooth muscle and gamma-actins in the pregnant rat myometrium. Biol. Reprod. 73, 773-780 (2005).

42. Nadeem, L. et al. Cytoplasmic mislocalization of p27 and CDK2 mediates the anti-migratory and anti-proliferative effects of Nodal in human trophoblast cells. J. Cell Sci. 126, 445-453 (2013).

43. Dong, X., Shylnova, O., Challis, J. R. \& Lye, S. J. Identification and characterization of the protein-associated splicing factor as a negative co-regulator of the progesterone receptor. J. Biol. Chem. 280, 13329-13340 (2005).

\section{Acknowledgements}

We thank Mrs Anna Dorogin and Ms Tamara Nedd-Roderique for their assistance in collecting and processing of mouse tissues and Mrs Anna Bucciarelli for the illustration in Fig. 7. This study was supported by a grant from Canadian Institute of Health Research (CIHR, MOP-111148) to S.L. and X.D.

\section{Author contributions}

S.L., X.D. and L.N. designed the project, L.N. performed research and analysed data, E.M.-Z. provided technical help, X.D. contributed plasmid constructs and PR stable cell lines, S.M. provided PR-inducible cell lines and intellectual input to the data interpretation, L.N., O.S. and S.L. wrote the manuscript. X.D. and S.L. are joint senior authors.

\section{Additional information}

Supplementary Information accompanies this paper at http://www.nature.com/ naturecommunications

Competing financial interests: The authors declare no competing financial interests.

Reprints and permission information is available online at http://npg.nature.com/ reprintsandpermissions/

How to cite this article: Nadeem, L. et al. Molecular evidence of functional progesterone withdrawal in human myometrium. Nat. Commun. 7:11565 doi: 10.1038/ncomms11565 (2016).

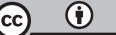

This work is licensed under a Creative Commons Attribution 4.0 International License. The images or other third party material in this article are included in the article's Creative Commons license, unless indicated otherwise in the credit line; if the material is not included under the Creative Commons license, users will need to obtain permission from the license holder to reproduce the material. To view a copy of this license, visit http://creativecommons.org/licenses/by/4.0/ 